\section{Minenopfer und Mine-Ex}

\section{H. Stirnemann}

Aranyaprathet 1992: Ein lebhaftes Städtchen in Thailand an der Grenze zu Kambodscha, mit einem Lager für 100000 kambodschanische Flüchtlinge, die vor dem Terror der Roten Khmer nach Thailand geflüchtet waren, und mit einem IKRK-Spital. Damals, nach mehr als 10 Jahren, begann die Repatriierung der Flüchtlinge. Viele von ihnen nahmen ihre Arbeit als Reisbauern wieder auf. Die Gefahr der unzähligen vergrabenen Minen war unterschätzt worden. Nacht für Nacht brachte man Minenverletzte über die Grenze nach Thailand zurück, weil es in Kambodscha damals noch keine funktionierenden Spitäler gab.

1992 hatte ich dort meine erste IKRK-Mission als Chirurg angetreten, voller Zuversicht, das nötige Fachwissen für meine Aufgabe mitzubringen. Das war auch der Fall, soweit es die chirurgische Technik betraf, und doch: Schon nach wenigen Tagen lag ich nachts übermüdet auf meinem Bett, suchte verzweifelt den nötigen Schlaf und fand ihn nicht. Warum? Bilder von zerfetzten Menschen mit abgerissenen Gliedern, durchlöcherten Augen, geschundenen Körpern standen vor meinen Augen, Bilder von Menschen, die mich als Arzt stumm und verzweifelt anschauten, auf Hilfe und Rettung hoffend, die ich ihnen nicht bringen konnte. Minenopfer; Tag für Tag; Nacht für Nacht.

Dort entstand meine Abscheu vor diesen Waffen. In langen Nächten wurde mir klar, dass dies nicht nur ein Problem der betroffenen Länder ist und dass die Lösung nicht allein Politikern und den humanitären Hilfswerken überlassen werden kann. Gleiches erlebte ich später in Pakistan und im Sudan.

\section{Dimensionen des Problems}

Dies sind keine Einzelfälle. Jeden Monat werden noch heute weltweit nach UN-Schätzungen etwa 2000 Menschen durch eine Mine verletzt oder getötet. Man nimmt an, dass mehr als 60 Länder mit Millionen Minen verseucht sind. Besonders betroffen sind: Mozambique, Angola, Ex-Jugoslawien, Afghanistan, Kambodscha, Iran, Irak, Vietnam [1].

Korrespondenz:

Prof. Dr. med. Hans Stirnemann

Grünaustrasse 7

CH-3400 Burgdorf
Folgen

- Verletzungen: Je nach Minenart (vergrabene Minen, Splitterminen, oft als Spielzeug getarnte sog. booby traps) werden nach Coupland drei Verletzungstypen unterschieden [2], am häufigsten sind traumatische Amputationen, ausgedehnte Körperverletzungen, Erblindung, Tod.

- Invalidität: Antipersonenminen sollen nicht töten, sondern ihren Opfern schwere Verletzungen zufügen mit dauernder Invalidität als Folge. Die Moral des Gegners wird damit untergraben und seine Infrastruktur maximal belastet.

Chirurgie kann an sich wenig tun für diese Verletzungen. Nach langem Spitalaufenthalt und oft mehreren Operationen verlassen über 50\% der Verwundeten, die das Glück hatten, ein Spital zu erreichen, es als dauernd Invalide, ohne nachfolgende staatliche Hilfe oder Unterstützung. Sie können sich nicht einmal Krücken leisten. Eine Reise in ein orthopädisches Zentrum ist oft ein grösseres Unternehmen, das die ganze Familie belastet. Arbeit finden sie keine, Betteln ist meistens das Einzige was ihnen bleibt.

Es genügt deshalb nicht, wenn wir diesen Menschen das Leben retten. Ohne Rehabilitationsmöglichkeiten beginnt die Leidenszeit für sie und ihre Familien erst nach Spitalaustritt.

Minen wurden bis vor wenigen Jahren (und heute immer noch in vielen Teilen der Welt) als «notwendige" und "legitime" Waffe betrachtet, obschon sie längst zum billigen und weitverbreiteten Terrorinstrument geworden sind. Sie verletzen elementares humanitäres Recht, weil sie ungezielt wirken («undiskriminiert"), unnötiges Leiden verursachen und mehrheitlich Zivilisten verletzen. In der Öffentlichkeit waren sie bis vor wenigen Jahren kein Thema. Das Problem wurde in westlichen Ländern verdrängt oder nicht wahrgenommen. Erst in letzter Zeit sind weltweit viele Menschen nicht mehr bereit, dieses Gemetzel weiterhin zu tolerieren und verlangen ein allgemeines Minenverbot.

\section{Der Ottawavertrag}

Nach langem politischem Ringen konnte im Dezember 1997 in 0ttawa ein Vertrag unterzeichnet werden, der zu einem absoluten Verbot für Entwicklung, Produktion, Lagerung, Handel und Anwendung von Antipersonenminen verpflichtet und auch eine Frist für die Entminungsaktionen setzt. 133 Länder haben ihn bis am 1. Januar 2000 unterzeichnet und 90 ratifiziert. Trotz dieser erfreulichen Entwicklung wird es noch Jahre und Jahrzehnte dauern, bis ein weltweites, allgemein gültiges und verbindliches Minenverbot bestehen wird. Und wenn dies einmal erreicht sein sollte, werden noch weitere Jahrzehnte verstreichen, bis der Boden der verseuchten Länder gesäubert ist. Solange wird es aber noch neue Minenopfer geben, um so mehr als wichtige Länder und gewisse Hauptproduzenten von Minen immer noch abseits stehen, z.B. USA, Russland, China, Pakistan, Indien etc. 
Unmittelbar vor und nach Unterzeichnung des Ottawavertrages waren die Medien und dadurch eine breite Öffentlichkeit für das Minenproblem sensibilisiert. Das führte zu einer erfreulichen Hilfswelle, die aber schon zwei Jahre später wieder abflaute. Die Welt beschäftigt sich mit anderen Problemen und anderen Katastrophen und die Minenopfer geraten wieder in Vergessenheit.

Dagegen kämpft Mine-Ex. Die Opfer sind noch über Jahre auf unsere Hilfe angewiesen. Die Bemühungen für ein Minenverbot dürfen nicht versanden.

\section{Das Mine-Ex-Projekt}

Nach meiner Rückkehr in die Schweiz verfolgte mich das Elend der Minenopfer. Im Kreise einiger Freunde des Rotary Club Burgdorf entstand die Idee eines Hilfswerks. Daraus entstand 1995 das Mine-Ex-Projekt in Partnerschaft mit dem IKRK, mit folgenden Zielen:

1. Hilfe für die Opfer (Prothesenherstellung),

2. Ausbildung von einheimischen Prothesenmachern,

3. Mithilfe im Kampf für ein weltweites, allgemeines Minenverbot.

Das Projekt hat sich inzwischen gut entwickelt. In der Schweiz wird es von vielen Rotaryclubs und einer sehr grossen Anzahl Sympathisanten unterstützt. Es hat sich auch auf das Ausland ausgeweitet. Unsere Hilfe aus der Schweiz geht nach Kambodscha, ein Land mit mehr als 25000 amputierten Minenopfern.

\section{Mine-Ex unterstützt:}

- das orthopädische Zentrum in des IKRK Battambang in West-Kambodscha. Hier werden mehr als 1000 der bewährten Polypropylenprothesen pro Jahr individuell angefertigt und angepasst unter Verwendung von Standardbauteilen (Stützelemente, Gelenke, Fuss). Jede Prothese hat eine begrenzte Lebensdauer und muss nach 2 bis 3 Jahren, bei Kindern nach 6 bis 12 Monaten, ersetzt werden;
- die Fabrik für Prothesenteile des IKRK in Phnom Penh. Sie ist zum Zentrum der Versorgung für das ganze Land geworden. Pro Jahr werden dort die Bauteile für etwa 7000 Prothesen hergestellt (dies entspricht dem Jahresbedarf von Kambodscha), und kostenlos an die fünf im Lande tätigen Hilfswerke verteilt, nämlich IKRK, AMCROSS (American Red Cross), CT (Cambodia Trust), H. I. (Handicap International), V. I. (Veterans International); - die Ausbildung von einheimischen Prothesenmachern, darunter Amputierte, die so einen sinnvollen Beruf erlernen;

- die Bemühungen des IKRK für ein totales, globales Minenverbot mit Seminaren, Vorträgen, Diskussionen, Artikeln, Anlässen, etc.

Seit 1997 unterstützt Mine-Ex die Werkstätten des IKRK in Kambodscha mit jährlich über Fr. 500 000.-. Anlässlich von regelmässig durchgeführten Informationsreisen (auf eigene Kosten) kann festgestellt werden, dass in beiden Betrieben durch motivierte Mitarbeiter (je 2 Europäer und 36 Einheimische) professionell, engagiert und rationell gearbeitet wird und dass die finanziellen Mittel exakt und im Sinne der Spender eingesetzt werden. Das IKRK ist auf breite finanzielle Unterstützung angewiesen, sollen Einrichtungen, die für die Minenopfer unentbehrlich und lebensnotwendig sind, kontinuierlich über mehrere Jahre funktionieren. Die betroffenen Länder können diese Aufgabe in der Regel weder technisch noch finanziell übernehmen.

Mine-Ex-Konto: UBS AG, Kirchberg, PC 30-35-9, zu Gunsten Mine-Ex, Kt. Nr. 235/7E677945.1

\section{Literatur}

1 Bottigliero I. 120 Million Landmines Deployed Worldwide: Fact or Fiction? Genève: Fondation Pro Victimis; 2000.

2 Coupland RM, Korver A.: Injuries from antipersonnel mines: the experience of the International Committee of the Red Cross. BMJ 1991;303:1501. 